\title{
INTERSTELLAR ABSORPTION OF SOME Be STARS
}

\author{
D. Briot \\ Observatoire de Paris, Paris, France \\ J. Zorec \\ Institut d'Astrophysique, Paris, France
}

\section{INTRODUCTION}

It is well known that the intrinsic colors of Be stars are not the same as those of "normal" B stars and they are often redder. So interstellar absorption of Be stars determined from photometric indices may be overestimated. In order to avoid this, a method often used to determine the interstellar absorption of Be stars is based on the ultraviolet interstellar absorption bump at $2200 \AA$ (Dorschner 1975; Nandy et al. 1975, 1976). However some peculiar Be stars exist whose circumstellar matter contributes to the $2200 \AA$ bump (Savage et al. 1978). The aim of this work is to test the deredenning method from the $2200 \AA$ bump for "classical" Be stars, in determining the interstellar absorption in the region of the Be star, using the surrounding normal stars.

\section{DESCRIPTION OF THE METHOD USED}

Calibration with normal $B$ stars

Throughout this study, the interstellar absorption will be expressed in the form of color excess $E(B-V)$. We first established a standard method of dereddening for normal B stars both from UBV photometric observations and from observations of the Balmer discontinuity (BCD method as defined in Chalonge and Divan, 1973) : the color excess thus obtained is noted $E(B-V) U B V+B C D$. Using this color excess, the $2200 \AA$ bump is calibrated, so defining a color excess : $E(B-V) 2200$. We then tested the surrounding star method for normal B stars. For each stars studied, a curve : $E(B-V)$ as a function of the distance, is established from the near surrounding stars (1ess than $2^{\circ}$ whenever possible and less than $5^{\circ}$ in the worst cases), data being taken in Deutschman et al. (1976). This allowed us to determine the interstellar absorption of the $B$ star studied : $E(B-V)$ sur. A good agreement is found between $E(B-V)$ sur and $E(B-V)_{U B V+B C D}$, as well as between $E(B-V)$ sur and $\mathrm{E}(\mathrm{B}-\mathrm{V})_{2200}$

\section{Application of this method to Be stars}

The sample studied here is made up of 56 Be stars for which we have enough information. We determined $E(B-V)_{2200}$ for Be stars in the same way we determined it for $B$ stars without emission. The determination of interstellar absorption from surrounding stars requires a good knowledge of the star distance and the determination of the distance is more difficult for a Be star than for a normal B star because of the uncertainties about the absolute magnitude of Be stars. The assumption was made that during a non-emission phase, absolute 
magnitude and color indices of Be stars are the same as those of $B$ stars without emission having the same spectral classification. The apparent magnitude $V$ corresponding to a non-emission phase of the Be star was determined, whenever possible, from the variations of the second Balmer discontinuity, and in the other cases, by extrapolation from the variations of the color indices $(U-B)$ and $(B-V)$.

\section{RESULTS AND DISCUSSION}

$E(B-V)$ sur VS. $E(B-V) 2200$, for the Be star sample studied here is plotted on figure 1 . We see in this diagram that the distribution is not symmetrical around the $45^{\circ}$ line but for most of the stars :

$E(B-V)_{2200} \leq E(B-V)$ sur. We recall that for $B$ stars : $E(B-V)_{2200} \cong E(B-V)$ sur. Is the result obtained here significant? The error in $E(B-V)$ sur comes mainly from the distance determination of Be stars. The error bars drawn on fig. 1 correspond to the estimation of this error $( \pm 0.5 \mathrm{mag}$. both on the absolute magnitude and on the $V$ magnitude during a phase without emission) and are not sufficient to justify the deviation from the $45^{\circ}$ line.

The star located at the top of the figure as $E(B-V)_{\text {sur }}>E(B-V)_{22} 20$ is HD 38010. However in Deutschman et al. (1976) there is no star close enough to this star. When closer surrounding stars from other catalogues are used, the plot corresponding to this star is located on the $45^{\circ}$ line, as shown by the dashed line. So it would appear that the star HD 38010 is located in a region where the interstellar absorption is less than in the neighbouring regions. This example shows how much the interstellar absorption as a function of the distance varies even in neighbouring regions. So it is important to use only very close surrounding stars in this type of study.

We looked for a correlation between the quantity $E(B-V)_{2200}-E(B-V)$ sur and, successively, the strength of emission, the spectral type, and the presence of a she11. No correlation would be found from the sample studied here.

As a conclusion, it would seem that, in the case of Be stars, the $2200 \AA$ bump is not only due to interstellar matter. But, the circumstellar matter forming the envelopes of Be stars contributes somewhat to this bump, by lines (FeII, FeIII, NiII, CrIII...) or by band absorption.

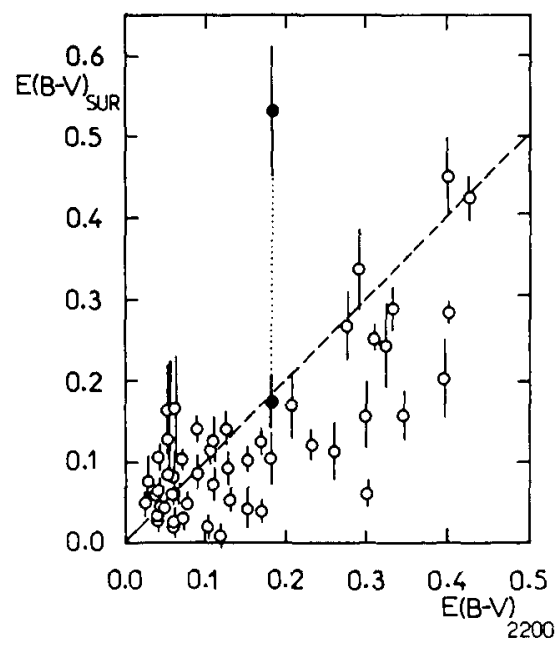

Figure 1. 


\section{REFERENCES}

Deutschman, W.A., Davis, R.J., Schild, R.E. (1976). Astrophys. J. Suppl. $30,97$.

Dorschner, J. (1975). Astrophys. Space Sci. 34, 39.

Chalonge, D., Divan, L. (1973). Astron. Astrophys. 23, 69.

Nandy, K., Thompson, G.I., Jamar, C., Monfils, A., Wilson, R. (1975).

Astron. Astrophys. 44, 195.

Nandy, K., Thompson, G.I., Jamar, C., Monfils, A., Wilson, R. (1976). Astron. Astrophys. 51, 63.

Savage, B.D., Wesselius, P.R., Swings, J.P., Thé, P.S. (1978). Astrophys. J. 224,149 .

\section{DISCUSSION FOLLOWING BRIOT}

Houziaux:

What happens when you determine again the distance of the Be star with the $\mathrm{E}(\mathrm{B}-\mathrm{V})_{\text {SUR }}$ ? Does the process converge?

Briot:

The process converges very quickly. Generally, after two or three iterations, you get differences of \pm 1 pc. 